\title{
Prevent, Detect, Respond, Mitigate Liquid Sodium Leakage, and Fire Accidents using AI
}

\author{
Praveen Sankarasubramanian, E. N. Ganesh
}

\begin{abstract}
Liquid /Fluid Sodium is very risky in nature[1]. It gets ignited thusly when introduced to air or water. They recall repressions for smothering administrators. Social wellbeing and affirmation are basic. In many impelled organizations and present-day nuclear force stations, liquid sodium utilized as a basic coolant. This technique offers challenges to nuclear powerhouses. The fire remains an overall supporter of intensity plants. Research on capable fire ID and cautioning structures has become a fervently discussed issue in ventures. In many force stations and manufacturing plants, PLC or microcontroller-based controllers are utilized. Most of the customary frameworks will be rule-based. Now and again, they give counterfeit cautions. There is a need to naturally recognize outpouring and fire utilizing most recent advances like man-made reasoning. These days, most of the enterprises are fixed with CCTV or other observing frameworks. Our point is to utilize Video and Traditional way to deal with distinguish and moderate fire. More secure force stations created utilizing authentic mishap information and encounters. This paper discusses liquid metal spillage disclosure frameworks and abbreviates its state of-data achievement. Different fire revelation techniques explored, and their characteristics and inadequacies are highlighted. An AI based methodology is suggested that will manage the fire recognizable proof. Proposed system consolidates new advances like Computer vision, CNN calculation with the customary sensor or micro-controller or PLC based application to identify the smoke and fire segments. Sensors recognize the spillages, spillages of the fluid metals. Computer Vision and CNN calculations recognizes the nearness of fire from a current video source. This will limit the opportunity of spillages and different dangers.
\end{abstract}

\section{Keywords: CNN, NUMPY, PYTHON, OPENCV, AI.}

\section{INTRODUCTION}

Sodium is an extremely hazardous in nature. [1][2][3] Responsive nature of the sodium makes it hard to recognize the purest type of this metal. Sodium is constituent of a few broadly utilized non-perilous parts like table salt, baking Soda and different meds.

The unadulterated metallic type of sodium

- Utilized in applications that require abnormal warmth move and electrical conductivity properties.

Revised Manuscript Received on May 27, 2020.

* Correspondence Author

Praveen Sankarasubramanian*, Research Scholar, Vels Institute of Science, Technology and Advanced Studies, (VISTAS), Chennai, Tamilnadu, India.

Dr. E. N. Ganesh, Dean School of Engineering, Vels Institute of Science, Technology and Advanced Studies, (VISTAS), Chennai, Tamilnadu, India.

(C) The Authors. Published by Blue Eyes Intelligence Engineering and Sciences Publication (BEIESP). This is an open access article under the CC BY-NC-ND license (http://creativecommons.org/licenses/by-nc-nd/4.0/)
- Significant metal for industries, fast breeder reactors and special applications.

- Utilized as an operator in handling different substances. It is an amazing reducing agent. It has a capacity to strip oxygen particles and different iotas from stable atoms. This substance response discharges large amount of energy and extra dangerous chemical by products.

- Hydrogen discharged as a gas. It detonates in air as hydrogen recombines with oxygen present in the encompassing climate.

- When detonates, it responds with oxygen destructive mixes like sodium oxide, sodium hydroxide

Fluid sodium exists in a scope of temperatures from softening point $98^{\circ} \mathrm{C}$ to breaking point $880^{\circ} \mathrm{C}$. Produce brilliant yellow light and thick smoke when consumed in air. On a sticky day, sodium responds with dampness noticeable all around; henceforth, they transferred in a sealed container. The sodium fire and smoke are destructive and harmful. They hinder the view. There are numerous examinations on sodium fire moderation and avoidance.

A few fluid metal spillage [38-39] location techniques proposed during the most recent decades. Spillage and fire recognition approach classified as exterior, interior, and biological.

Different sensor based leakage and fire detection are discussed in the papers [10-37], and summary of those sensing methods are marked in the table 1.

Table- I: Existing Detection Methods

\begin{tabular}{|c|l|}
\hline $\begin{array}{c}\text { DETECTION } \\
\text { METHOD }\end{array}$ & \multicolumn{1}{|c|}{ DETECTED USING } \\
\hline EXTERIOR & $\begin{array}{l}\text { Sound / acoustic sensors } \\
\text { Ground penetration sensors and radars } \\
\text { Vapour sampling } \\
\text { Fibre optic sensors } \\
\text { Infrared thermography } \\
\text { Fluorescent chemicals. } \\
\text { Electromechanical impedance } \\
\text { Capacitance } \\
\text { Accelerometer }\end{array}$ \\
\hline INTERIOR & $\begin{array}{l}\text { Negative pressure } \\
\text { Pressure point analysis } \\
\text { Digital signal processing } \\
\text { Dynamic modeling }\end{array}$ \\
\hline
\end{tabular}

Published By:

Blue Eyes Intelligence Engineering

\& Sciences Publication

(C) Copyright: All rights reserved.

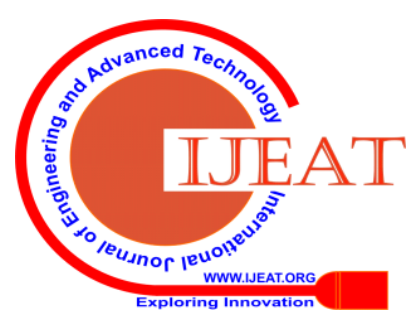




\begin{tabular}{|c|l|}
\hline $\begin{array}{c}\text { VISUAL \& } \\
\text { BIOLOGICAL }\end{array}$ & $\begin{array}{l}\text { Trained humans and dogs } \\
\text { Visual based bolt and joints monitoring }\end{array}$ \\
\hline REMOTE & Autonomous Underwater Vehicles \\
MONITORING & $\begin{array}{l}\text { Drones } \\
\text { Helicopters }\end{array}$ \\
\hline OTHER & $\begin{array}{l}\text { Computational } \\
\text { Non-technical } \\
\text { Non-continuous } \\
\text { Software } \\
\text { Auditory } \\
\\
\text { Hardware }\end{array}$ \\
\hline
\end{tabular}

Fire location framework is a significant part in the surveillance framework. Fire is one of the most significant dangers in a framework. It makes serious harm human life, properties, and enterprises. Fire identification frameworks assume a significant job in a surveillance framework. Beginning period fire identification expected to forestall the wildfire. Fire discovery frameworks consistently screens Indoor situations. Different sensors recognize the event of fire and dependent on its choices are taken. The unwavering quality of the fire recognition relies on cautious determination and situating of the sensors. A few sensors are not appropriate for open spaces. Ordinary fire identification frameworks endure fire detecting and correspondence delay. In this investigation, various frameworks are grouped, and their upsides and downsides examined.[10-37]

\section{RELATED WORK}

In the year, 1984 Gracie et al [1] inferred that there is no exact ignition temperature value for sodium. They presumed that a sodium oxide film on a sodium pool influences the ignition temperature. Nevertheless, the ignition temperature has been the focal point of many researches. Numerous investigates done to comprehend the unconstrained ignition component. Gaurav Yadav et al, [4] proposed picture-handling procedures to distinguish the fire. The calculation identifies the outright estimations of the RGB organizes. Picture portioned. The calculation allows a straightforward limit-based standard. Red worth, Ratio of Red to Green and Red to Blue - distinguished. Shading identification framework applies the particular property of the RGB organizes and gives the yield result as a picture with chosen zone of shading discovery. Dey, Papan and Das, Subir \&Das, [5] proposed a microcontroller-based fire dousing framework. Microcontroller based fire stifling framework is a dependable methodology. This framework is pertinent to various sizes of fire quenchers and high controlling ability over them. The plan is straightforward, and it permits least support work. This framework has no moving parts and bogus caution decreased. Precision and security of the fire sensor utilized, microcontroller's speed timing granularity and IO include Improves the general execution of microcontroller-based fire dousing framework. Prevalence of the microcontroller-based framework is expanding gradually, and it replaces the more established variant of fire quenching frameworks. He, Bingsong and Zhao et al [6], proposed a DSP calculation model-based plan for fire discovery framework. The fire discovery calculation was planned utilizing Simulink and C code. Mahesh et al proposed a video-based fire-disturbing framework. Picture arrangements procured from a video source. Picture sources changed over as casings and had the option to distinguish the fire from the closer view of the picture outline. The proposed approach had a few constraints. It cannot recognize the seething fire. Execution of the application was poor because of the shrouded fires (thick smoke) Therefore, the proposed strategy can possibly identify if fire is plainly obvious to the camera. In 2008, Setiawan, Joga et al [7], proposed firefighting robot that utilizes the advantages of computer-generated reality and pictures the movement of the firefighting robot. The proposed model gives a sensibly exact systematic portrayal of the fire. In the year 2018, Dunnings and Breckon [8] proposed a continuous decreased intricacy profound convolutional neural system engineering to identify the fire pixel district in video or picture outline. They proposed a hearty ongoing fire location calculation with $93 \%$ precision for recognizing fire from entire picture and $89 \%$ exactness with super pixel confinement system. Unpredictability of the framework diminished with legitimate determination of the system engineering. These decreased designs expanded the computational presentation by three - multiple times. It presents to 17 FPS preparing on the contemporary equipment free of the fleeting data. X. Lang, P. Li, Z. Hu, H. Ren and Y. Li [9], proposed a LSTSVM (LEAST SQUARES TWIN SUPPORT VECTOR MACHINES) calculation to distinguish the non-fixed and nonlinear qualities of the signs to find the whole position and perceive the diverse break openings. Wavelet investigation used to get better element extraction. Highlights extricated with time space attributes and waveform qualities under various working conditions. Parameters like component vector, total mean worth, powerful worth, and kurtosis, top worth factor of the contribution under the working condition. For improving, the precision of the time postpone estimation and evacuate every recurrence band of clamor wavelet investigation and LMD calculation utilized. The proposed technique can get higher precision of the time postpone and diminish the area blunder.

\section{ARTIFICIAL INTELLIGENCE AND MACHINE LEARNING}

Conventional methods replaced by IoT due to the rapid developments in Machine Learning, IoT and Artificial Learning methods. Image processing techniques with the traditional sensor approach will provide a robust fire identifying methodology. High-resolution cameras detect the motion of fire pixels, sodium droplets, and edge of the fire/flame and measure the area of the flame. The proposed paper uses the image sequence acquired from the video. Proposed an Adaptive learning fire detection methodology subtracts the foreground moving flame from the background objects. Rules applied to identify the color of the fire and to determine whether the detected foreground object is fire or not. Upcoming section discusses the different AI techniques employed in the Fire and leakage detection.

This paper proposes a novel method of fire detection by processing image sequence

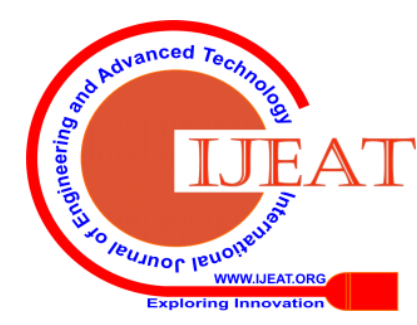


acquired from a video. The proposed video-based fire-detection system uses adaptive background subtraction to detect foreground moving objects and then verified by the rule-based fire color model to determine whether the detected foreground object is a fire or not. YCbCr color space used to model the fire pixel classification. In addition to the motion and color, the detected fire candidate regions analyzed in temporal domain to detect the fire flicker. Some Morphological operations used to enhance the features of detected fire candidate regions. All the above clues are combining to form the fire detection system. The performance of the proposed algorithm tested on a set of videos comprising the fire, fire colored object and non-fire. The experimental results show that the proposed system is highly successful in detecting fire and /or flames.

\section{METHODOLOGY}

Video documents comprise of frames. Frames are the principal element of the video. Rate of these frames are more than the human's perception of vision. Frames acquired from a live video feed, changed over into pictures. Various methods utilized to change over video as frames and pictures. For instance- frame2im utilized in MATLAB.

Basic fire discovery approach was composed utilizing python, OPENCV and NUMPY. The methodology works by taking a video and preparing it outline by outline. It switches the shading space to the HSV (Hue, Saturation and Value). This HSV is progressively like the way people see shading than RGB space. Next higher and lower shading esteems are characterized. A cover (Threshold extend) characterized to part the fire/fire hues from the foundation outline. [4]

To separate fire from the foundation, picture pressure, transforming, picture division, edge division and highlight extraction of the fire district is applied.

Picture segmentation done to part the forefront picture and the background picture. "Picture edge discovery strategy" used to identify the shading difference. "Numerical techniques" used to identify the brilliance and edge of the picture. Threshold value is set to remove the object from the background.

In the component extraction process, all information pixels taken and wanted pixels assembled.

Set of features will extract the desired fire pixel from the image pixel

- Automated smoke detection using multiple detectors

- Flame detection using Python and Image detection

- Convolution Neural Network for Real time Fire Detection

- Fire detection using Raspberry Pi, Python and Open CV

\section{A. Detection of fluid sodium fire using OPENCV and NUMPY:}

Video captured to detect the fluid sodium fire and converted as pictures. Gaussian Blur Algorithm applied to blur the picture. In a "BGR color space", fire pixel found between [13, 50, and 50] and [35, 255, 255]. This range applied on the captured video and at the same time, the image transformed in HSV color space. Figure 1 shows the fire image and Figure 2 shows the output of the algorithm.

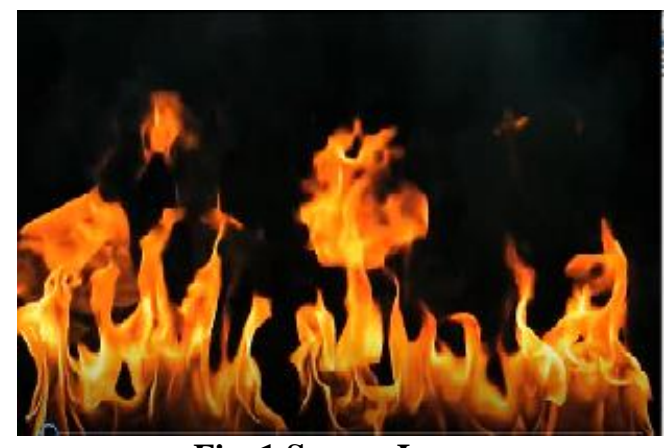

Fig. 1.Source Image

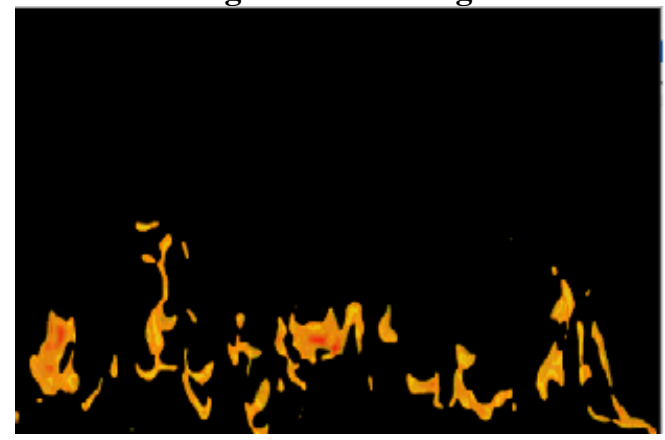

Fig. 2.Output Image

This approach mainly concentrates on color-based segmentation. Hence, the probability of the error rate will be higher.

1) Steps for detecting the fire from a video:

- Import OpenCV and NumPy Package

- Get the Video input.

- Initialize upper and lower color value to detect the fire from the image

- Iterate the video and get frames.

oApply Gaussian Blur on the frames to differentiate foreground and background image.

oApply HSV Transform on the blurred image

o Mask the HSV Transformed image with the upper and lower fire value.

oPerform a BITWISE AND OPERATION on the input frame, HSV Transformed value, and the MASK and show the final output.

oRepeat the above steps until final no frame is present

\section{B. Detection of fluid sodium fire using CNN and Deep Learning:}

To improve the accuracy and quality of the captured result CNN and Deep Learning are used. Depth wise separable Convolution Neural Networks are more efficient, require less power, can perform better than the standard convolution method.[8]

This method uses the frameworks like Load TENSORFLOW, KERAS, PYIMAGESEARCH, and NUMPY. First, the training data set is loaded with a collection of fire and non-fire pictures. This random collection iterated in a loop. Image resized in various dimensions like $128 * 128$ and predictions performed.

Published By:

Blue Eyes Intelligence Engineering

\& Sciences Publication

(C) Copyright: All rights reserved.

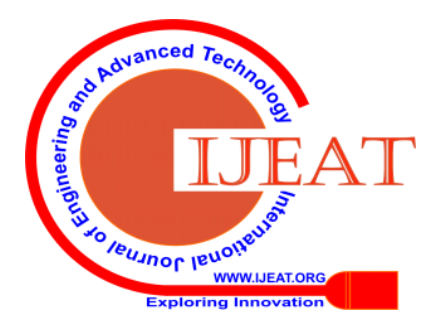


1) Steps for detecting the fire from a video:

- Import OpenCV and Tensor Flow framework.

- Get the Video input.

- Get the video properties such as length, width, height.

- Iterate through the video frames

oResize the images for better prediction

$\circ$ Apply the resized image with the pretrained model

oSlice the frames and find the contour of the fire and apply a bitwise operator to on the frame and the mask. Apply this frame to the pretrained model.

oAbove two masking principle accurately identifies the fire in the video.

o Apply these steps till end of the video is reached.

\section{CHALLENGES}

This proposed application gives a false output if an image has impression of nightfall in a water body. This picture will have fell shades of red and orange shades; consequently, it makes an inferno impact. In those circumstances, fire discovery will be an issue. The proposed application utilized in indoor areas and this could improve the presentation of the application. The preparation informational collection ought to be as tremendous as could reasonably be expected and gathering the informational index will be a test.

\section{RESULTS}

500 sample videos were taken for testing. Results demonstrated that the discovery of fire utilizing OPENCV and NUMPY gave 81 percent of precision. At the point when it was gotten together with the complex CNN calculations, its precision was increased to 93 percent. Henceforth use of proposed fire identification system gives us a predominant structure execution regarding less fake caution and as such a higher system execution is practiced. Precision can similarly be moreover extended by applying various viable count in every time of area. Additionally, the structure is logically reliable in reefing the result comes out from existing area techniques.

\section{CONCLUSION}

Different fluid metal fire identification methods were examined, and its condition of-information accomplishment were outlined. This paper proposed a novel technique for fire location by handling picture succession procured from a video. Robust fire recognizing system proposed by joining the conventional sensor approach with the picture preparing methods. The Error rate of the framework is decreased by presenting CNN calculations and 93\% accuracy achieved with this approach. Exactness can likewise be expanded by applying distinctive productive calculations in each period of recognition.

\section{REFERENCES}

1. Gracie, J.D. and Droher, J.J., "A Study of Sodium Fire," NAA-SR-4383 (1960).

2. Akita, K. and Yamashika, S., Report of Fire Research Institute, No.24, and pp.38-43 (1964) In Japanese.

3. Yuasa, S., Proc. Twentieth Symposium (International) on Combustion, the Combustion Institute, pp.1869-1876 (1984).

4. Gaurav Yadav et al / Indian Journal of Computer Science and Engineering (IJCSE) OPTIMIZED FLAME DETECTION USING IMAGE PROCESSING BASED TECHNIQUES

5. Dey, Papan \& Das, Subir \& Das, Choton \& Ahmed, Toufiq \& Reza, C M F S \& Rahman, Mahbub. (2014). Design and Implementation of an Automatic Fire Extinguishing System Based on Fault Secure Multi-Detectors.

6. He, Bingsong \& Zhao, Xueping \& Zhou, Zhiguo \& Fan, Zheyi. (2013). Implementation of a Fire Detection Algorithm on TMS320DM642 DSP using MATLAB/Simulink. 10.2991/iccnce.2013.155.

7. Setiawan, Joga \& Subchan, Mochamad \& Budiyono, Agus. (2008). Virtual Reality Simulation of Fire Fighting Robot Dynamic and Motion CoRR. abs/0804.3882. 10.1007/978-3-642-00264-9 12.

8. Dunnings and Breckon, In Proc. International Conference on Image Processing IEEE, 2018

9. X. Lang, P. Li, Z. Hu, H. Ren and Y. Li, "Leak Detection and Location of Pipelines Based on LMD and Least Squares Twin Support Vector Machine," in IEEE Access, vol. 5, pp. 8659-8668, 2017.URL: snumber=7859429

10. Meng, L.; Yuxing, L.; Wuchang, W.; Juntao, F. Experimental study on leak detection and location for gas pipeline based on acoustic method. J. Loss Prev. Process Ind. 2012, 25, 90-102.

11. Jin, H.; Zhang, L.; Liang, W.; Ding, Q. Integrated leakage detection and localization model for gas pipelines based on the acoustic wave method. J. Loss Prev. Process Ind. 2014, 27, 74-88.

Mahmutoglu, Y.; Turk, K. A passive acoustic based system to locate hole in underwater natural gas pipelines. Digit. Signal Process. 2018, 76, 59-65. sensors for monitoring pressure and stiffness changes in out-of-round pipes. Struct. Control Health Monit. 2016, 23, 303-314.

. Jia, Z.; Ren, L.; Li, H.; Sun, W. Pipeline Leak Localization Based on Sci. 2018, 8, 146

15. Png, W.H.; Lin, H.S.; Pua, C.H.; Rahman, F.A. Pipeline monitoring and leak detection using Loop integrated

Mach Zehnder Interferometer optical fiber sensor. Opt. Fiber Technol. 2018, 46, 221-225.

Ni, S.; Huang, Y.; Lo, K.; Lin, D. Buried pipe detection by ground penetrating radar using the discrete wavelet transform. Compute Geotech. 2010, 37, 440-448.

. Hoarau, Q.; Ginolhac, G.; Atto, A.M.; Nicolas, J. Robust adaptive 293-305.

19. Li, H.; Xiao, D.; Zhao, X. Morphological filtering assisted Fifield-pipeline small leakage detection. In Proceedings of the 2009 IEEE International Conference on System, Man and Cybernetics, SMC 2009, San Antonio, TX, USA, 11-14 October 2009; pp. 3769-3774.

20. Delgado, M.R.; Mendoza, O.B. A comparison between leak location methods based on the negative pressure wave. In Proceedings of the 14th International Conference on Electrical Engineering, Computing Science , on negative pressure wave method. Process Saf. Environ. Prot. 2018, 119, 181-190.

22. Kam, S.I. Mechanistic modeling of pipeline leak detection at fixed inlet

Tian, S.; Du, J.; Shao, S.; Xu, H.; Tian, C. A study on a real-time leak pressure and flow rate. Appl. Therm. Eng. 2016, 95, 462-470. simulating the entire leaking process and calculating the liquid leakage volume of a damaged pressurized pipeline. J. Hazard. Mater. 2017, 332, 19-32.

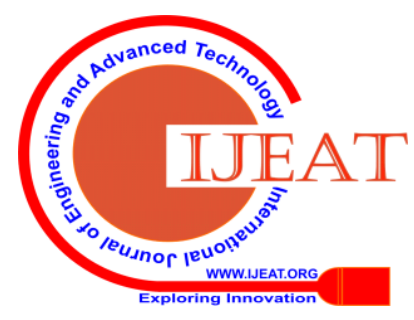


25. Yang, Z.; Fan, S.; Xiong, T. Simulation and Numerical Calculation on Pipeline Leakage Process. In Proceedings of the 2010 2nd International Symposium on Information Engineering and Electronic Commerce (IEEC), Ternopil, Ukraine, 23-25 July 2010; IEEE: Piscataway, NJ, USA, 2010; pp. 1-5.

26. Li, X.; Chen, G.; Zhang, R.; Zhu, H.; Fu, J. Simulation and assessment of underwater gas release and dispersion from subsea gas pipelines leak. Process Saf. Environ. Prot. 2018, 119, 46-57.

27. Wan, J.; Yu, Y.; Wu, Y.; Feng, R.; Yu, N. Hierarchical leak detection and localization method in natural gas pipeline monitoring sensor networks. Sensors 2012, 12, 189-214.

28. Manekiya, M.H.; Arulmozhivarman, P. Leakage detection and estimation using IR thermography.

29. In Proceedings of the 2016 International Conference on Communication and Signal Processing (ICCSP), Melmaruvathur, India, 6-8 April 2016; IEEE: Piscataway, NJ, USA, 2016; pp. 1516-1519.

30. Sun, J.; Peng, Z.; Wen, J. Leakage aperture recognition based on ensemble local mean decomposition and sparse representation for classification of natural gas pipeline. Measurement 2017, 108, 91-100.

31. Gao, Y.; Liu, Y.; Ma, Y.; Cheng, X.; Yang, J. Application of the differentiation process into the correlation-based leak detection in urban pipeline networks. Mech. Syst. Signal Process. 2018, 112, 251-264.

32. Yin, S.; Weng, Y.; Song, Z.; Cheng, B.; Gu, H.; Wang, H.; Yao, J. Mass transfer characteristics of pipeline leak-before-break in a nuclear power station. Appl. Therm. Eng. 2018, 142, 194-202.

33. Turner, N. Hardware and software techniques for pipeline integrity and leak detection monitoring. Soc. Pet. Eng. 1991, 139-148.

34. Murvay, P.; Silea, I. A survey on gas leak detection and localization techniques. J. Loss Prev. Process Ind. 2012,25, 966-973.

35. Zhang, J. Designing a cost-effective and reliable pipeline leak-detection system. Pipes Pipelines Int. 1997, 42, 20-26.

36. Geiger, G.; Vogt, D.; Tetzner, R. State-of-the-art in leak detection and localization. Oil Gas Eur. Mag. 2006,32, 1-26.

37. Golmohamadi, M. Pipeline Leak Detection. Master's Thesis, Missouri University of Science and Technology, Rolla, MO, USA, 2015.

38. Praveen Sankarasubramanian,"Effective Handling of Fluids and Liquid Metals using IoT","International Journal of Institution of Safety Engineers (India)", Volume 3, Issue 1, Jan-March 20

39. Praveen Sankarasubramanian, E.N. Ganesh, "IoT Based Prediction for Industrial Ecosystem", "International Journal of Engineering and Advanced Technology (IJEAT)”, ISSN: 2249-8958, Volume-8 Issue-5, June 2019

\section{AUTHORS PROFILE}

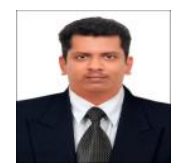

Praveen Sankarasubramanian D.ECE, M.Tech, PGDBA, AMIE(India), CEng(India), MICSES, SMISE(India) is a Research Scholar, Vels Institute of Science, Technology and Advanced Studies,(VISTAS), Chennai. He as a tech-savvy software developer who has an IOSH certification. He got state first in 10th Tamil Language and He received a Gold Medalist with 600/600 mark in Diploma. He completed BE in Government College of Engineering, Tirunelveli and finished his M.Tech (Software Systems - Data Analytics) from Birla Institute of Technology and Science, Pilani, Rajasthan. Mail id: praveengrb@gmail.com

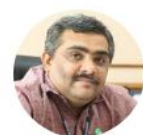

Dr. E. N. Ganesh Dean School of Engineering, Vels Institute of Science, Technology and Advanced Studies, (VISTAS), Chennai. He is a Professor in the field of Electronics for the past 20 years, Specialized in Nano electronics and Microelectronics. His research work in Quantum electronics adjudged the best thesis and received a Gold medal in Ph.D. He has finished M.Tech from IIT Madras in Microelectronics. He has 54 Conferences and Journal Publications. Mail id: enganesh50@gmail.com.

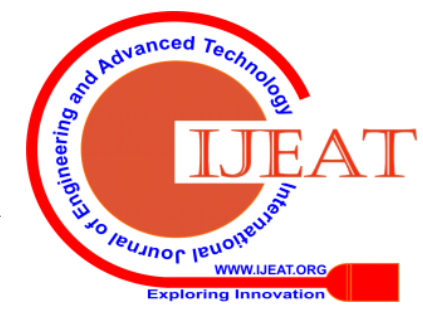

